\title{
Controlling the Change in the Shape of a Cylindrical Shell Subjected to External Hydrostatic Pressure
}

\author{
Vladimir V.Kiselev and Dennis V.Dolgikh \\ M.N. Mikheev Institute of Metal Physics of the Ural Branch of the Russian Academy of Sciences, \\ Yekaterinburg, Russia \\ Email: kiseliev@imp.uran.ru
}

\begin{abstract}
Analytic and numerical analysis for the initial non-linear elastic stage of changing in the shape of a circular shell subjected to a high-pressure liquid is performed, with the shell being under two rigid constraints: an external cylindrical cavity and/or an internal rod. In this stage, the emergence of alternating bulges and depressions is governed by the balance between the nonlinearity and dispersion effects. In the framework of the Cosserat theory, the dependence of the curvature of the shell cross-section on the external pressure is obtained. Knowing the curvature make it possible to restore the form of the cross section with the methods of the differential geometry. It is shown that unwanted wave-like folds and rigid ribs on the deformed shell surface can be eliminated by suitably selecting the constraints. Cost-efficient ways of producing hollow articles from pipe billets with the hydrostatic pressure method are discussed.
\end{abstract}

Keywords: Cylindrical shell, deformation, hydrostatic pressure, non-linear theory of elasticity

\section{Introduction}

The ways of controlling the evolution of local instabilities of the hydrostatically compressed metal shells were studied experimentally $[1,2]$. The ranges for the deformation degrees of the shell material, external pressure values and geometrical parameters of the shell were established for the cases when an initially circular shell takes a new circular, square or tooth-like cross-section shape under pre-assigned rigid constraints. These results open the possibility of developing new technologies for obtaining hollow articles with complex shapes from tube billets with minimal time, energy and material costs. The linear theory gives only a rough estimation of the critical loads leading to losing the shell shape stability and of a possible number of the waves emerged [3,4]. To date, almost all the experimental and theoretical investigations were aimed at searching for the conditions when each shell retains its shape unchanged under pre-assigned external impacts. This is extremely important for calculations and design of many vital constructions such as submarine hulls, pipes for drilling oil wells, high-speed rail cars, etc.

At the same time, the effects of strongly non-linear elastic bendings of the shell and the ways of controlling the change in its shape remain hardly investigated. The majority of the studies concerning the change in the shell shape use numerical modeling without applying analytical methods (see $[5,6,7,8]$ and references therein). The most substantial results are obtained for pressurized shells made from a rubber-like material [6]. However, the numerical approach does not explain fully the regularities of the phenomena observed and never investigates the ways of controlling the change in the shape of the samples. A combination of both numerical and analytical approaches is thought to be more effective. The problem can be constructively solved in the framework of simplified non-linear models. On the one hand, the latter take correctly into account the basic interactions and rigid constraints governing the change in the shell shape, and on the other hand, admit exact solutions. Analysis of the solutions gives criteria for choosing the constraints to drive the process of change in the shell shape.

In the experiments, on the surface of the shell subjected to hydrostatic pressure, bulges and depressions alternating in the circular direction arise. At the same time, the shell retains its cross-section unchanged along the generating line. Therefore, the nature of the process of corrugating the shell can be understood within the problem on change in the shape of an initially circular thin ring of unit height. In such a formulation, the equations that determine non-linear bends of the ring coincide with the equations describing the change in the shape of a flexible rod, with its axis residing in a fixed plane. Beginning 
from Euler's works, the theory of flexible rods serves as a reference for solving boundary problems for elastic systems subjected to given loads. It is appropriate to recall some results of the theory of elastic rods, useful for discussing the problem of change in the shell shape.

It is known that the rods can transform their shape when undergone by varied external loads. Though some types of equilibrium of the rods and rings are known to be unstable, they can be stabilized by introducing confirmatory constraints [3]. It is such an approach that applies to controlling the change in the shell shape $[1,2]$.

When the rod is compressed rapidly, i.e. the load-increase time is shorter than the deformation relaxation time, its elements cannot move immediately in the direction normal to the rod axis. Before the bends of the rod become visible, the compressing impact can reach not only a first critical value but higher ones typical for different stationary states of the rod. In this case, the final states of the loaded rods with higher forms of the bends are observed. Such a situation was first described within the linear theory of elasticity [9]. As in the case of the elastic rods, the hydrostatically compressed shells also have bulges and depressions on their surface $[1,2,3]$. The number of such formations depends on ratio of geometric and material parameters of the shell and the magnitude of the external pressure.

The problems of change in shape of rods and rings under different point loads or mutual contacts of rings and smooth surfaces attract still intent attention of researchers $([10,11,12]$ and the references therein). However, the influence of the constraints on localization of bends of a hydrostatically compressed shell in the initial non-linear elastic stage of deformation, the ways of controlling the process of change in the shell shape are not yet studied. The papers $[13,14]$ show analytic solutions for the Cosserat model, describing large non-linear bends of the cross-section of the shell under hydrostatic pressure. It is demonstrated that under certain conditions the shell cross-section can be shaped into regular polygons even in absence of external constraints. The formation of the plane facets on the surface of the loaded shell is important for technological applications and can be observed in experiments [3]. The traditional theory of shells does not explain how they form.

The present paper develops, generalizes the approach mentioned above and analyses Cosserat-type models by analytical and numerical methods for describing significant deformation of a cylindrical circular shell subjected to hydrostatic pressure, under two rigid constraints: an outer bounding cylindrical cavity and a round rod inside the latter. Typical scenarios of localization of the shell deformations during the initial non-linear elastic stage of the change in its shape are revealed. The work reports on the conditions (shell geometry and appropriate range of external pressures) under which the external constraints prevent from forming wave folds and rigid ribs on the deformed shell surface. The general principles of producing hollow articles with varying cross-sections from pipe billets are formulated.

\section{Formulation of the Model}

Let the length of a circular cylindrical shell subjected to hydrostatic compression be a few times greater than its radius. Then, the longitudinal deformation of the middle segment of the shell is assumed to be the same at each step of changing its (transverse) cross-section $[15,16]$. Consider a circular ring of unitheight in the middle segment of the shell. We examine the dependence of its two-dimensional equilibrium states on applied external pressure $p$. Suppose the axis of the shell to be directed along the $O z$-axis of a Cartesian coordinate system.

In the non-deformed state, the middle line of the cross-section of the shell has radius $R$. When deformation occurs, the line can be described by the curve

$$
\mathbf{r}(s)=(x(s), y(s), 0),
$$

where $s$ is the natural parameter of the curve, measured off from some point of the middle line. Then, the unit tangent vector of the curve is

$$
\boldsymbol{\tau}=\frac{\mathrm{d} \mathbf{r}}{\mathrm{d} s} .
$$

In the main approximation, when bended, the shell saves the distances between points of the middle line. So we have $0 \leq s \leq 2 \pi R$. Let $\mathbf{k}=(0,0,1)$ be the unit vector directed along the axis of the shell. Then, 
the unit vector of the outward normal to the shell surface is defined by

$$
\mathbf{n}=[\mathbf{k} \times \boldsymbol{\tau}]
$$

Cosserat's approach assumes that the interaction of parts of the shell surface is carried out by forces and bending moments distributed along the lines of their separation [15]. Let us consider a small part of the ring corresponding to the interval $[s, s+\mathrm{d} s]$. The material of the ring situated in the region $\tilde{s} \geq s+\mathrm{d} s$ affects this part of the ring with the resultant contact force $\mathbf{q}(s+\mathrm{d} s)$ and the resultant contact couple $\mathbf{m}(s+\mathrm{d} s)=m(s+\mathrm{d} s) \mathbf{k}$. Whereas the material situated in the region $\tilde{s} \leq s$ affects the part with the resultant force $-\mathbf{q}(s)$ and the bending moment $-\mathbf{m}(s)=-m(s) \mathbf{k}$. The hydrostatic pressure always acts along the normal vector. Thus, the element under consideration is affected by the force $p \mathbf{n} d s$. In the case of an external pressure we have $p<0$. The equilibrium conditions for the ring element reduce to Eqs. $[15]$

$$
\frac{\mathrm{d} \mathbf{q}}{\mathrm{d} s}+p \mathbf{n}=0, \quad \frac{\mathrm{d} \mathbf{m}}{\mathrm{d} s}+[\boldsymbol{\tau} \times \mathbf{q}]=0 .
$$

If the shell is thin enough, the Euler-Bernoulli-Clebsch constitutive equation can be employed to describe the moment $m[10,11,12,17]$ :

$$
m=-\gamma\left(\tilde{\varkappa}+\frac{1}{R}\right)
$$

where $\gamma$ is a coefficient of proportionality. The approximation (5) coincides with the relation of the traditional theory of geometrically non-linear elastic shells under the condition $\tilde{\varkappa} / h \ll 1[3,11,17]$. Here $\tilde{\varkappa}$ is the curvature of the shell cross section and $h$ is its thickness. The linear theory of shells gives

$$
\gamma=\gamma_{0} h^{3}, \quad \gamma_{0}=\frac{E}{12\left(1-\nu^{2}\right)},
$$

$E$ is Young's modulus, $\nu$ is Poisson's ratio. The physical nonlinearity of the material, in particular its elastic-plastic properties, is often taken into account by renormalizing the elastic moduli [3]. Further, we regard $\gamma_{0}$ as a phenomenological parameter of the model. Using the approximation (5) we are going to obtain exact solutions of the problem and establish the key features of deforming the shell in the presence of constraints. As a result, we will reveal the possibility of controlling the change in the shape of shells subjected to the external hydrostatic compression.

The vector of the contact force $\mathbf{q}$ lies in the $x O y$ plane and, therefore, admits the representation

$$
\mathbf{q}=q_{n} \mathbf{n}+q_{\tau} \boldsymbol{\tau} .
$$

Taking into account (6) and Frenet-Serret formulae

$$
\frac{\mathrm{d} \boldsymbol{\tau}}{\mathrm{d} s}=\tilde{\varkappa} \mathbf{n} ; \quad \frac{\mathrm{d} \mathbf{n}}{\mathrm{d} s}=-\tilde{\varkappa} \boldsymbol{\tau},
$$

from (4) and (5), we derive a closed set of differential equations to calculate $q_{n}, q_{\tau}, \tilde{\varkappa}$ :

$$
\frac{\mathrm{d} q_{\tau}}{\mathrm{d} s}-q_{n} \tilde{\varkappa}=0, \quad \frac{\mathrm{d} q_{n}}{\mathrm{~d} s}+p+q_{\tau} \tilde{\varkappa}=0, \quad \gamma \frac{\mathrm{d} \tilde{\varkappa}}{\mathrm{d} s}+q_{n}=0
$$

Hence, the shear and tangential forces can be expressed in terms of the curvature of the shell cross-section:

$$
q_{n}=-\gamma \frac{\mathrm{d} \tilde{\varkappa}}{\mathrm{d} s}, \quad q_{\tau}=\gamma\left(Q-\frac{\tilde{\varkappa}^{2}}{2}\right) .
$$

The integration constant $Q$ will be determined below. The curvature of the middle line of the shell cross-section satisfies the equation

$$
\frac{\mathrm{d}^{2} \tilde{\varkappa}}{\mathrm{d} s^{2}}=Q \tilde{\varkappa}-\frac{|p|}{\gamma}-\frac{\tilde{\varkappa}^{3}}{2} .
$$

For the further analysis, it is convenient to use the dimensionless variables

$$
\theta=s / R, \quad \varkappa=R \tilde{\varkappa}, \quad q_{0}=R^{2} Q .
$$


Thus, $0 \leq \theta \leq 2 \pi$ and the first integral of Eq. (10) acquires the form

$$
\left(\frac{\mathrm{d} \varkappa}{\mathrm{d} \theta}\right)^{2}=q_{0} \varkappa^{2}-\frac{2|p|}{\gamma_{0}}\left(\frac{R}{h}\right)^{3} \varkappa-\frac{\varkappa^{4}}{4}+\frac{l_{0}}{4},
$$

where $l_{0}$ is yet another integration constant.

Analysis shows, that the solutions of Eq. (11) may be physically meaningful, i.e. be unambiguous and with no self-intersection loops, if the 4-th degree polynom on the right-hand side of Eq. (11) has only two real roots $a$ and $b$ and two complex-conjugate roots $c=b_{1}+\mathrm{i} a_{1}$ and $c^{*}=b_{1}-\mathrm{i} a_{1}$. The roots are related to the parameters of Eq. (11) as follows:

$$
\begin{gathered}
b_{1}=-\frac{1}{2}(a+b), \quad l_{0}=-a b|c|^{2}, \\
4 q_{0}=\frac{3}{4}(a+b)^{2}-a_{1}^{2}-a b, \\
\frac{8|p|}{\gamma_{0}}\left(\frac{R}{h}\right)^{3}=-(a+b)\left[a_{1}^{2}+\frac{1}{4}(a-b)^{2}\right]>0,
\end{gathered}
$$

where $b<a<-b$ The parameter $b$ is always negative. The desired solution of Eq. (11) has the form [18]

$$
\varkappa=\frac{a B(1-\operatorname{cn} \chi)+b A(1+\operatorname{cn} \chi)}{B(1-\operatorname{cn} \chi)+A(1+\operatorname{cn} \chi)}
$$

where $\chi=\theta / 2 g, A^{2}=\left(a-b_{1}\right)^{2}+a_{1}^{2}, B^{2}=\left(b-b_{1}\right)^{2}+a_{1}^{2}, g=(A B)^{-1 / 2}$. The modulus of the Jacobi function $k$ is given by the expression

$$
k^{2}=\frac{(a-b)^{2}-(A-B)^{2}}{4 A B}, \quad 0 \leq k^{2} \leq 1
$$

The values of the curvature of the middle line must be the same at both $\theta=0$ and $\theta=2 \pi$. This requirement leads to the first limitation imposed on the solution parameters:

$$
\pi \sqrt{A B}=4 K(k) m
$$

where $K(k)$ is the complete elliptic integral of the first kind, the parameter $m=2,3,4 \ldots$ specifies the number of the bulges on the shell cross-section.

At the initial stage of change in the shell shape, forming patterns of the bulges on the surface begins when the magnitude of pressure exceeds a critical threshold value. This process is governed by both the nonlinearity and dispersion effects. The nonlinear interactions result in localizing of the deformations and the effects of dispersion contribute to spreading of such peculiarities. Every equilibrium state of the shell is possible within a certain range of the pressure. The lower the pressure the fewer number of the bulges in the cross section of the shell is possible. The nonlinearity of the problem is that the ranges of the pressure, each corresponding to its own number of bulges, can overlap each other $[13,14]$.

The most general approach to the analysis of changing in the shape of loaded rods is considering a set of possible static configurations and studying bifurcations between each other with energetic principles (see $[3,15]$ and references therein). For the shell under the hydrostatic pressure quantity

$$
\left(\frac{\mathrm{d} \varkappa}{\mathrm{d} \theta}\right)^{2}-q_{0} \varkappa^{2}+\frac{2|p|}{\gamma_{0}}\left(\frac{R}{h}\right)^{3} \varkappa+\frac{\varkappa^{4}}{4}=-\frac{1}{4} a b|c|^{2}
$$

corresponds to the density of the energy. Hence, the value of the pressure $p_{\mathrm{c}}$ for which switching from the state $m$ to the state $m+1$ is possible is determined by the condition

$$
-\left.a b|c|^{2}\right|_{p=p_{\mathrm{c}, m}}=-\left.a b|c|^{2}\right|_{p=p_{\mathrm{c}, m+1}} .
$$




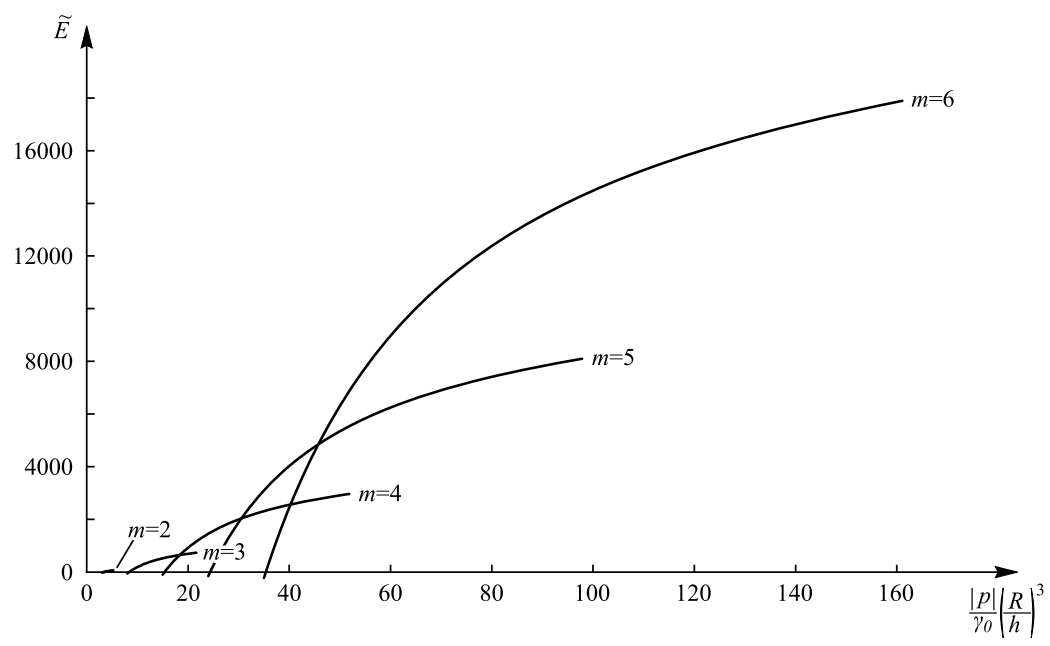

Figure 1. The pressure ranges for the equilibrium state with the various numbers $m$ of the bulges on the shell surface $\left(\tilde{E}=-a b|c|^{2} / 4\right)$.

Analysis shows, that the pressure range for $m=2$ doesn't overlap with the range for $m=3$ (Fig. 1). Therefore, switching from one such a state to another one is impossible.

Switching from the $m=3$ state to the $m=4$ state is quite possible under relatively low external pressure $p_{\mathrm{c}}$. For the shell having four bulges on its surface or more switching to next state can occur under higher magnitude of the external pressure. As the pressure grows, a sequence of switches throughout a number of consecutive states can take place. If the rate of pressure growth is low the shell consecutively switches from the state $m=3$ throughout the states $m=4,5,6, \ldots$ At the same time, if the rate of the pressure growth is high enough the shell can immediately switch to the state $m=6$ after having switched from the $m=3$ state to the $m=4$ one. Fig. 1 demonstrates that in the latter case the full increments of the pressure and the energy of the system are lesser than in the former one.

The positive definite second variation of the energy density (17) is the condition of sufficiency for the stability of the stationary state $\varkappa=\varkappa_{0}$. The configuration $\varkappa_{0}$ is stable if

$$
\frac{3}{2} \varkappa_{0}^{2}-q_{0}>0
$$

This inequality is equivalent to the condition

$$
\frac{2|p|}{\gamma_{0}|a+b|}\left(\frac{R}{h}\right)^{3}+\frac{1}{4}\left(5 a^{2}-b^{2}\right)>0 .
$$

Analysis shows, that inequality (19) is fulfilled within the pressure range for every configuration with the parameter $m=2,3,4,5$.

From (15) it follows, that the curvature $\varkappa(\theta) \in[b, a]$. The parameter $b$ is always negative, as mentioned above. Values of $\theta$ such that $\varkappa(\theta)=b(p)$ correspond to the crests of the bulges clearly visible on the shell surface. Depending on the pressure magnitude, the parameter $a$ can be both negative and positive. The parameters $\{\theta \mid \varkappa(\theta)=a(p)<0\}$ correspond to the points of the bulge crests whose amplitude is smaller than for the points $\{\theta \mid \varkappa(\theta)=b(p)\}(0>a>b)$. The parameters $\{\theta \mid \varkappa(\theta)=a(p)>0\}$ correspond to the points of the depressions which arise as the pressure magnitude grows (Fig. 2). The case $a(p)=0$ is of great interest. For such pressure the facets emerge on the shell surface, with the cross-section of the shell looking like a regular polygon with smoothed vertices (Fig. 3)

The unit vectors $\mathbf{n}$ and $\boldsymbol{\tau}$ lie in the $x O y$ plane and can be parametrized as

$$
\mathbf{n}=(\cos \Phi(\theta), \sin \Phi(\theta)), \quad \boldsymbol{\tau}=(-\sin \Phi(\theta), \cos \Phi(\theta)) .
$$

The Frenet-Serret formulae (8) can be reduced to the equation for the phase

$$
\frac{\mathrm{d} \Phi}{\mathrm{d} \theta}=-\varkappa(\theta) .
$$




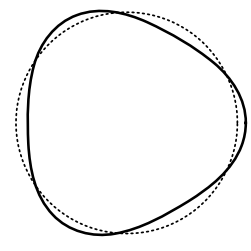

$a<0$

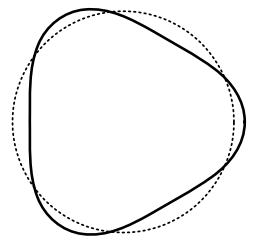

$a=0$

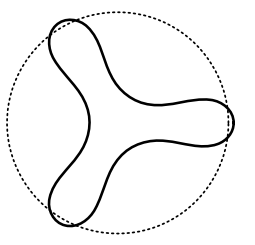

$a>0$

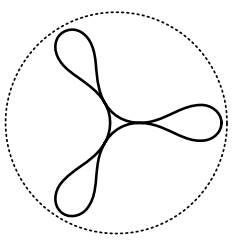

Figure 2. The shell cross-sections for different values of the external pressure, $m=3$

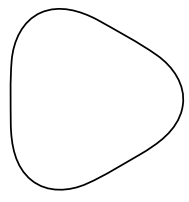

$m=3$

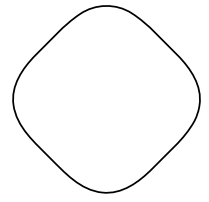

$m=4$

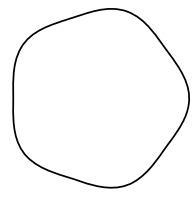

$m=5$

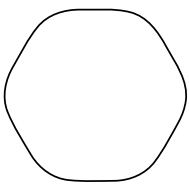

$m=6$

Figure 3. Configurations of the shell with facets for $m=3,4,5,6$

The solution of Eq. (21) has the form $[13,14]$

$$
\begin{aligned}
\Phi(\theta)=\left[\frac{A B}{a+b}-\frac{(a B+b A)}{A+B}\right] \theta- & \\
& -\frac{(A+B)^{2}}{2 \sqrt{A B}(a+b)} \Pi\left(\frac{\theta \sqrt{A B}}{2}, \alpha^{2}, k\right)+ \\
& +2 \operatorname{acrtan}\left[\frac{(a-b)}{2 \sqrt{A B}} \operatorname{sd}\left(\frac{\theta \sqrt{A B}}{2}, k\right)\right]
\end{aligned}
$$

and the property $\Phi(\theta=0)<\Phi(\theta=2 \pi)$ is valid. Here

$$
\operatorname{sd}(x, k)=\frac{\operatorname{sn}(x, k)}{\operatorname{dn}(x, k)},
$$

$\Pi\left(x, \alpha^{2}, k\right)$ is the complete elliptic integral of the third kind [18]

$$
\Pi\left(x, \alpha^{2}, k\right)=\int_{0}^{x} \frac{\mathrm{d} x^{\prime}}{1-\alpha^{2} \operatorname{sn}^{2}\left(x^{\prime}, k\right)}
$$

with the modulus $k$ and the parameter $\alpha^{2}$

$$
\alpha^{2}=-\frac{(A-B)^{2}}{4 A B}<0
$$

The phase $\Phi(\theta)$ is measured off from a ray in the $x O y$ plane, the position of which is determined by a constant, appearing after integration of Eq. (21). In (22) the integration constant is chosen in such a way, that $\Phi(\theta=0)=0$, i. e. the ray coincides with the $O x$ axis of the Cartesian coordinate system and passes through the bulge crest of the cross section.

The function $\Phi(\theta)$ must get increment of $2 \pi$ as variable $\theta$ varies from 0 to $2 \pi$ for the curve $\mathbf{r}(\theta)$ not to have self intersections. This requirement gives one more restriction for the parameters of the problem:

$$
\frac{A b+B a}{A+B}+1+2 m[\Lambda(\beta, k)-1]=0,
$$


where $\Lambda(\beta, k)$ is the Heuman lamba-function [18]:

$$
\begin{gathered}
\Lambda(\beta, k)=\frac{2}{\pi}\left[E(k) F\left(\beta, k^{\prime}\right)+K(k) E\left(\beta, k^{\prime}\right)-K(k) F\left(\beta, k^{\prime}\right)\right], \\
\beta=\arcsin \frac{2 \sqrt{A B}}{A+B}, \quad k^{\prime}=\sqrt{1-k^{2}}
\end{gathered}
$$

$F\left(\beta, k^{\prime}\right)$ and $E\left(\beta, k^{\prime}\right)$ are the incomplete elliptic integrals of the first and second kind respectively with the modulus $k^{\prime}$.

Integration of Eqs. (2), (20) gives the shape of the middle line of the shell section:

$$
x=x_{0}-R \int_{0}^{\theta} \sin \Phi(\theta) \mathrm{d} \theta, \quad y=y_{0}+R \int_{0}^{\theta} \cos \Phi(\theta) \mathrm{d} \theta .
$$

The integration constants

$$
x_{0}=\frac{R}{2 \pi} \int_{0}^{2 \pi} \int_{0}^{\theta} \sin \Phi\left(\theta^{\prime}\right) \mathrm{d} \theta^{\prime} \mathrm{d} \theta, \quad y_{0}=-\frac{R}{2 \pi} \int_{0}^{2 \pi} \int_{0}^{\theta} \cos \Phi\left(\theta^{\prime}\right) \mathrm{d} \theta^{\prime} \mathrm{d} \theta
$$

are chosen from the condition that the shell axis coincides with the $O z$ one.

Generally, constraints (16), (23) and (14) constitute a closed system of equations for calculating the values $a, b, a_{1}$ as functions of the pressure magnitude $p$ and the parameter $m$.

The model proposed describes significant non-linear deformations of the shell. The approximation of the linear theory corresponds to a small change in the cross section curvature if the parameters $a$ and $b$ are close to one another and to the curvature of the non-deformed cross section:

$$
a=-1+\varepsilon, \quad b=-1-\varepsilon, \quad 0<\varepsilon \ll 1 .
$$

In this case, from (12) it follows that

$$
b_{1} \approx 1, \quad c \approx 1+\mathrm{i} a_{1} .
$$

Hence

$$
A \approx B \approx \sqrt{4+a_{1}^{2}}, \quad k \approx 0 .
$$

As the result of it, all the significant relations can be resolved into the elementary functions. Constraint (23) is satisfied as an identity, constraint (16) results in

$$
A \approx B \approx \sqrt{4+a_{1}^{2}}=2 m
$$

Low amplitude corrugation of the cross section is given by $[13,14]$

$$
\sqrt{x^{2}+y^{2}} \approx R\left(1+\frac{\varepsilon \cos m \theta}{m^{2}-1}\right)
$$

According to (14), (26) corrugation of the shell starts as the external pressure overcome the threshold value

$$
|p|_{\text {lin }}=\gamma_{0}\left(\frac{h}{R}\right)^{3}\left(m^{2}-1\right)
$$

If we use Eq. (6) for the parameter $\gamma_{0}$, then the threshold value (28) coincides with the critical load for the linear theory [3].

In $[13,14]$ the possible equilibrium states of the shell depending on the value of the external pressure were analytically described. In this paper the influence of the most simple constraints on corrugation of the shell is investigated. Applying such constraints can eliminate unwanted folds and patterns on the shell surface, which arise on the first non-linear elastic stage of deformation. 


\section{Deformation under Constraints}

Here we will analyse peculiarities of deformation of the initially circular shell confined by a number of constraints by the external hydrostatic pressure. To begin with, let us consider the deformation of the shell placed into a hollow circular arbor. Then, we will discuss the case of the shell around an inner rigid rod.

Each of the cases has its own disadvantages which prevent from producing articles of the variable diameter along the shell axis. At the same time, useful properties of the methods considered below complement one another. Therefore, the combined application of these constraints deliver possibility for controlling the process of changing in the shell shape under the hydrostatic pressure.

\subsection{The Shell inside a Hollow Cylindrical Arbor}

Let the shell with the radius $R$ be placed into the hollow cylindrical arbor with the radius $R^{(\mathrm{e})}$, with $R<R^{(\mathrm{e})}$. The axes of the shell and the arbor coincide and are directed along the $O z$ axis. Being under the external pressure the shell will lose the stability of the form once the magnitude of the pressure overcomes some threshold value. Then, $m$ bulges stretched along the shell generatrix will arise on the surface. Suppose the radius $R^{(\mathrm{e})}$ relates to the radius $R$ in a such way, that the shell touches the arbor at the points $A$ and $E$ (see Fig. 4) as $p=p_{\mathrm{e}}$. From that moment the shell is adjacent to the arbor along the $\operatorname{arcs} A B$ and $E D$. As long as the symmetry of the shell stays intact during the interaction of

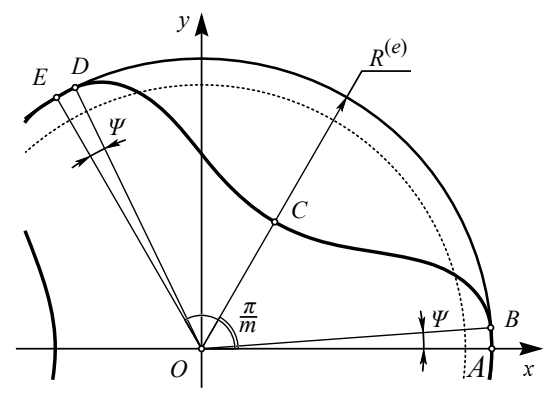

Figure 4. The scheme of changing in the shape of the shell constrained by the outer arbor

the shell with the arbor, the full representation of the process of deformation is given by the segment $\left[s_{A}, s_{E}\right]=[0,2 \pi R / m]$.

Let the angle $\psi$ specify the arcs of the outer arbor which the parts of the cross section $E D$ and $A B$ are adjancent to. As long as the length of the $E D$ arc equals to the length of the $A B$ arc, the natural parameter $s$ of the free part of the shell $B C D$ varies within $\left[s_{B}, s_{D}\right]$, where

$$
s_{B}=R^{(\mathrm{e})} \psi, \quad s_{D}=\frac{2 \pi R}{m}-R^{(\mathrm{e})} \psi .
$$

For the further analysis it is more convenient to use the dimensionless variables

$$
\begin{aligned}
& \theta=\frac{s}{R}, \quad \varkappa=R \tilde{\varkappa}, \quad x=\frac{\tilde{x}}{R}, \quad y=\frac{\tilde{y}}{R}, \\
& \xi=\frac{R^{(\mathrm{e})}}{R}, \quad \theta_{B}=\xi \psi, \quad \theta_{D}=\frac{2 \pi}{m}-\xi \psi .
\end{aligned}
$$

Here $\tilde{\varkappa}$ is the initial curvature of the shell cross section, $\tilde{x}, \tilde{y}$ are the dimensional Cartesian coordinates of the $\mathbf{r}(s)$ radius vector in the cross section plane $x O y$.

As before, the phase $\Phi(\theta)$ is defined by the equation

$$
\Phi(\theta)=-\int_{\theta_{B}}^{\theta} \varkappa\left(\theta^{\prime}\right) \mathrm{d} \theta^{\prime}+\Phi_{\mathrm{c}}
$$


with $\Phi_{\mathrm{c}}$ being the integration constant. The dimensionless coordinates for the radius vector of the free part of the middle line can be written out as follows

$$
x(\theta)=x_{0}-\int_{\theta_{D}}^{\theta} \sin \Phi\left(\theta^{\prime}\right) \mathrm{d} \theta^{\prime}, \quad y(\theta)=y_{0}+\int_{\theta_{B}}^{\theta} \cos \Phi\left(\theta^{\prime}\right) \mathrm{d} \theta^{\prime},
$$

where $x_{0}, y_{0}$ are the integration constants. Let $\left(x_{D}, y_{D}\right)$ and $\left(x_{B}, y_{B}\right)$ be the coordinates of points $D$ and $B$ respectively. The condition $x\left(\theta_{D}\right)=x_{D}$ determines the constant $x_{0}$

$$
x_{0}=\xi \cos \left[\frac{2 \pi}{m}-\psi\right] \text {, }
$$

and the condition $y\left(\theta_{B}\right)=y_{B}$ determines the constant $y_{0}$ :

$$
y_{0}=\xi \sin \Psi .
$$

The part $A B C D E$ is a smooth curve. At the $B$ point the tangent vector $\boldsymbol{\tau}(\theta)$ coincides with the tangent vector for the arbor. It results in the condition for the integration constant $\Phi_{\mathrm{c}}$ :

$$
\Phi_{\mathrm{c}}=\Phi\left(\theta_{B}\right)=\Psi
$$

Let us denote the phase function $(22)$ as $\Phi_{0}(\theta)$. Then (29) takes the form

$$
\Phi(\theta)=\Phi_{0}(\theta)-\Phi_{0}\left(\theta_{B}\right)+\Psi
$$

Similarly, the condition for the tangent vectors coincidence at the $D$ point results in

$$
\Phi\left(\theta_{D}\right)=\frac{2 \pi}{m}-\psi
$$

The first equation for calculation of the parameters $a, b, a_{1}, \psi$ results from matching (31) with (32):

$$
\Phi_{0}\left(\theta_{D}\right)-\Phi_{0}\left(\theta_{B}\right)=\frac{2 \pi}{m}-2 \psi
$$

The second equation results from the equality $x_{B}=x\left(\theta_{B}\right)=\xi \cos \psi$ :

$$
\xi \cos \psi=\xi \cos \left[\frac{2 \pi}{m}-\psi\right]+\int_{\theta_{B}}^{\theta_{D}} \sin \Phi\left(\theta^{\prime}\right) \mathrm{d} \theta^{\prime}
$$

And the third one is the result of the equation $y_{D}=y\left(\theta_{D}\right)=\xi \sin (2 \pi / m-\psi)$ :

$$
\xi \sin \left[\frac{2 \pi}{m}-\psi\right]=\xi \sin \psi+\int_{\theta_{B}}^{\theta_{D}} \cos \Phi\left(\theta^{\prime}\right) \mathrm{d} \theta^{\prime}
$$

As in the case for the unconstrained shell, the fourth Eq. (14) relates the parameters $a, b, a_{1}$ with the magnitude of the external pressure $p$.

Eqs. (33), (34), (35), (14) form a system of transcendent equations for the unknown values $a, b, a_{1}, \psi$ with the parameters $m, p, \xi$. The system has solution for any $p \in\left[p_{\mathrm{e}}, p_{\text {lim }}\right]$, with the limit pressure $p_{\text {lim }}$ depending on the relative radius of the outer arbor $\xi=R^{(\mathrm{e})} / R$ for every natural number $m>1$ (see Fig. 5).

Analysis shows, that deformation of the shell constrained by the outer arbor has distinctive peculiarities, which persist for every number of the bulges $m$. After the shell has come in the contact with the arbor the bulges adjoin the arbor tighter as the pressure grows. Then, this process becomes slower and slower. The shell gets a "rigid" form. Growth of the pressure slightly changes the shell (Fig. 6).

Consequently, the outer arbor not only constrains the amplitude of the bulges on the shell surface but stabilizes their form throughout a wide range of the pressure. At the same time, the outer arbor doesn't set any required profile for the shell cross section. 


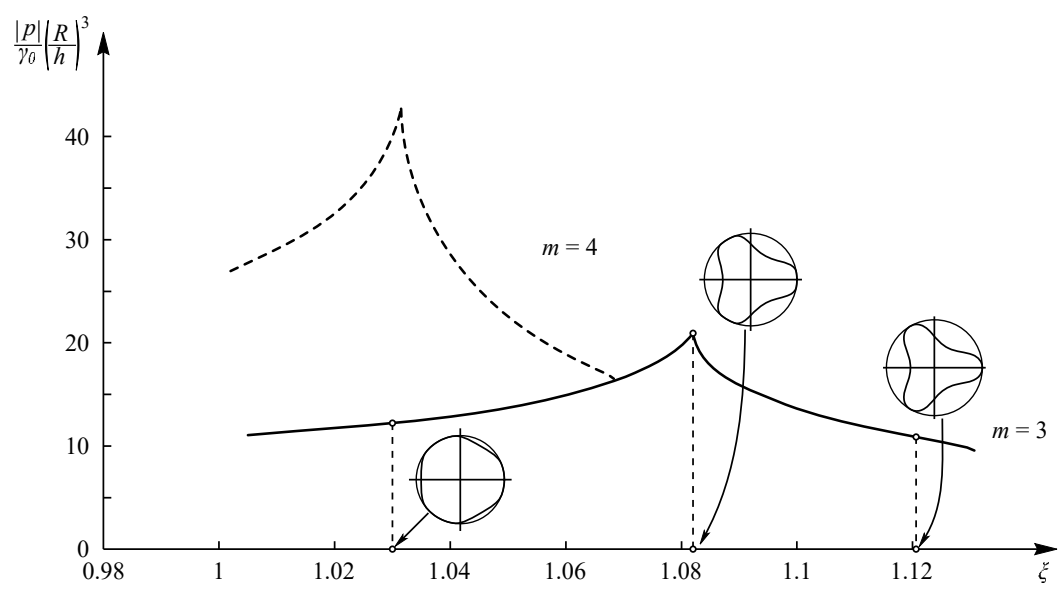

Figure 5. The limit pressure $p_{\text {lim }}$ dependence on the parameter $\xi=R^{(e)} / R$. The small insertions demonstrate the final states of the cross section for the different $\xi$ and the corresponding limit magnitudes of the pressure

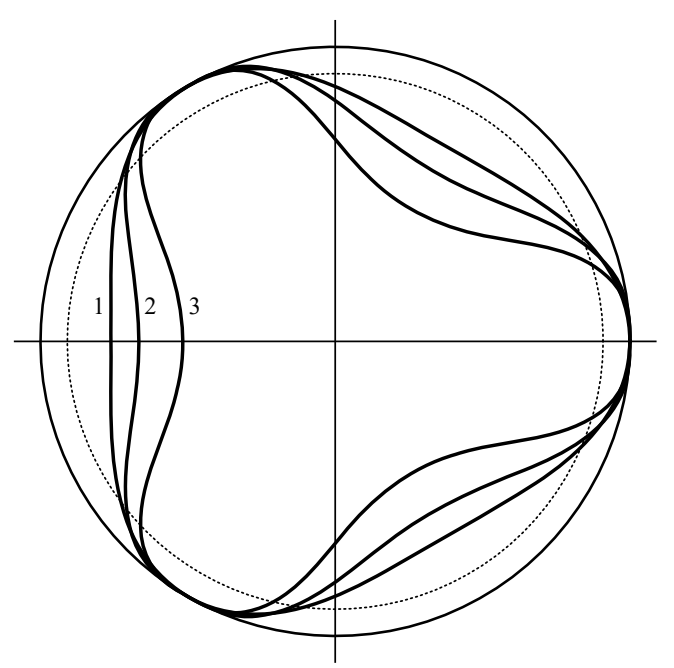

Figure 6. The evolution of the cross section shape of the constrained shell for $\xi=1.1: 1-$ the moment of the contact; 2 - some intermediate configuration; 3 - the limit state 
It is important for applications that the internal stresses get concentrated in the shell along the shell arcs adjoining to the arbor near the bulges vertices. The compressive stresses in the shell material along the directions $\boldsymbol{\tau}(\theta)$ are given by the formula

$$
T=\frac{q_{\tau}}{h}=\gamma_{0}\left(\frac{h}{R}\right)^{2}\left(q_{0}-\frac{\varkappa^{2}}{2}\right) .
$$

The shear stresses along the directions $\mathbf{n}(\theta)$ have the form

$$
N=\frac{q_{n}}{h}=-\gamma_{0}\left(\frac{h}{R}\right)^{2} \frac{\mathrm{d} \varkappa}{\mathrm{d} \theta} .
$$

For $m=3$ the dependence of the maximum stress intensity $S=\sqrt{N^{2}+T^{2}}$ on the pressure magnitude is shown on Fig. 7. The magnitude $S$ reaches the maximum at the vertex of the convexity (the point $B$ on Fig. 4).

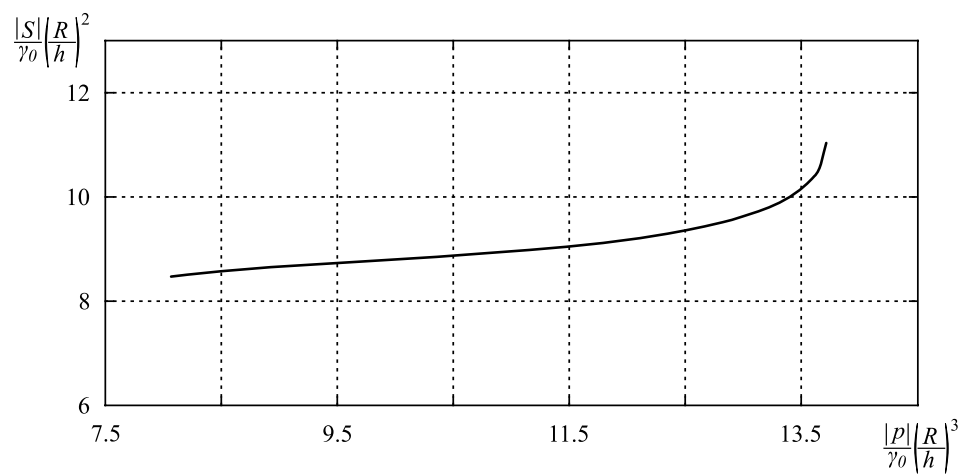

Figure 7. The dependence of the maximum stress intensity $S$ on the pressure $p$ for the shell constrained by the outer arbor $(m=3, \xi=1.05)$

\subsection{The Change in the Shape of the Shell on a Rigid Rod}

Let us place a rigid round rod of the radius $R^{(\mathrm{i})}$ inside the shell $\left(R^{(\mathrm{i})}<R\right)$. In Fig. 8 the deformed shell comes into contact with the rod at the point $B$ (the common axis of the shell and the rod coincides with the $O z$ one).

Provided the arc length $s$ of the cross section measured off from the point $A$, the point $B$ corresponds to the parameter $s_{B}=\pi R / m$ or the dimensionless parameter $\theta_{B}=s_{B} / R=\pi / m$.

As the pressure is increased further, the shell begins to bend around the rigid rod. The point where the shell and the rod touch each other is shifted from the point $B$ to the point $C$. The natural parameter $s_{C}=R^{(\mathrm{i})} \varphi+\pi\left(R-R^{(\mathrm{i})}\right) / m$ or the dimensionless parameter $\theta_{C}=\delta \varphi+\pi(1-\delta) / m, \delta=R^{(\mathrm{i})} / R$ corresponds to the point $C$. Here $\varphi$ is the angle between the $O x$ axis and the radial line $O C$ (see Fig. 8).

For $s \in\left[s_{A}, s_{C}\right]$ the shape of the shell is defined by the phase $\Phi(\theta)(22)$. The parameters $a, b, a_{1}, \varphi$ are determined by additional equations.

From the condition for the tangential vector $\tau$ to the surface of the shell section to coincide with the tangential vector to the surface of the rigid rod at the point $C$ we obtain

$$
\Phi\left(\theta_{C}\right)=\varphi .
$$

The coordinates of points of the line $A C$ are found by integrating Eqs. (2) and (20)

$$
\begin{aligned}
& x(\theta)=R \int_{\theta}^{\theta_{C}} \sin \Phi\left(\theta^{\prime}\right) \mathrm{d} \theta^{\prime}+R^{(\mathrm{i})} \cos \varphi, \\
& y(\theta)=R \int_{0}^{\theta} \cos \Phi\left(\theta^{\prime}\right) \mathrm{d} \theta^{\prime} .
\end{aligned}
$$




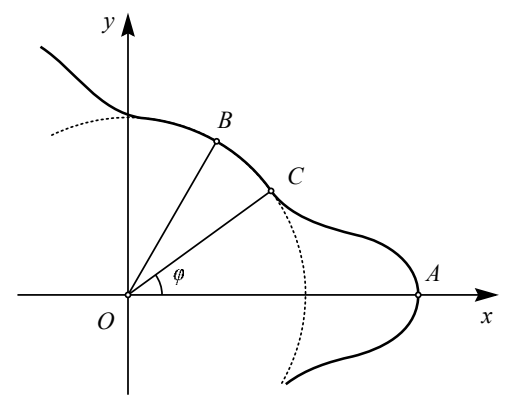

Figure 8. Changing in the shell shape around the inner rod

At the point $C$ the coordinates of the shell coincide with the coordinates of the rod. The thickness of the shell is neglected for simplicity. This requirement is equivalent to the equation

$$
\int_{0}^{\theta_{C}} \cos \Phi\left(\theta^{\prime}\right) \mathrm{d} \theta^{\prime}=\delta \sin \varphi
$$

Along the arc where the shell adjoins to the rod, the external pressure is compensated by the reaction of the rod along the direction $\mathbf{n}$. Hence, at the point $\mathrm{C}$ we have

$$
q_{n}=\left.\gamma_{0}\left(\frac{h}{R}\right)^{2} \frac{\mathrm{d} \varkappa}{\mathrm{d} \theta}\right|_{\theta=\theta_{C}}=0
$$

i. e. $\varkappa\left(\theta_{C}\right)=a$. It is equivalent to

$$
\frac{\pi}{m}(1-\delta)+\delta \varphi=\frac{4 K(k)}{\sqrt{A B}}
$$

Eqs. (36)-(38), (14) define the parameters $a, b, a_{1}, \varphi$ as functions of $|p|$ and $m$.

Fig. 9 shows several solutions that illustrate the change in the shape of the shell on the rigid rod under the external pressure. The left figure represents the moment of contact for the shell and the rod, the right figure represents the limit state of the shell.
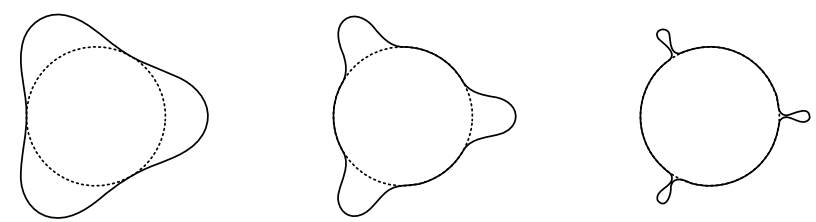

Figure 9. Changing in the shape of the shell around the rigid rod, $m=3$

When the difference in the radii of the inner rod and the shell is great, then rigid ribs are formed on the side surface of the shell, which are not removed even when the pressure increases further. Generally, using only an inner rod does not always allow to reshape the shell as intended.

\subsection{The Shell under Two Constraints}

The main goal is processing tube billets into hollow articles of variable diameter along the shell axis by the hydrostatic pressure.

In principle, presence of the round rod inside the shell allows to decrease the radius of the cross section. However, the rigid ribs remain on its surface. 
Introduction of an additional bounding cylinder allows not only to stabilize the shape of the shell with the wave-like profile but also control changing in the shape near the smoothed crests on the shell surface within some range of the pressure. This opportunity is only available, if the geometric parameters of the experimental device are fit in such a way, that the shell being deformed first touches the inner rod and after that the outer cylinder. Below we will analyse the change in the shell shape under two constraints within the framework of the proposed model.

Let a rigid rod of the radius $R^{(\mathrm{i})}$ be placed inside the shell, and the shell be placed inside a hollow cylinder of the radius $R^{(e)}$, with $R^{(i)}<R<R^{(e)}$ and axes of the rod, the shell and the cylinder coinciding with the $O z$ axis (Fig. 10). In a certain sense this case is a combination of the previous ones.

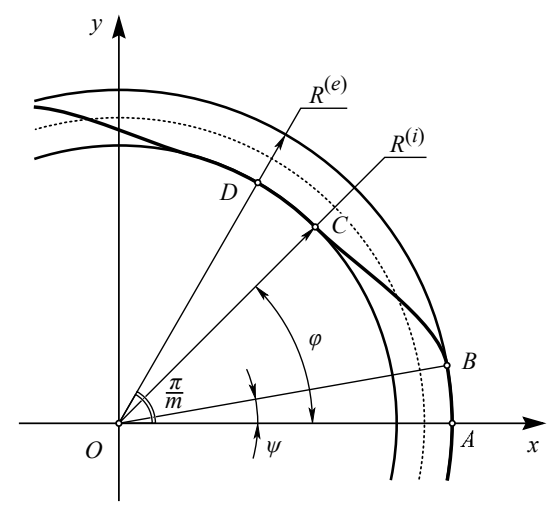

Figure 10. The scheme of changing in the shell shape under two constraints

As the pressure $p$ is increased further, the shell changes in its shape, gets $m$ bulges. After the pressure $p$ having reached a certain value $p_{\mathrm{i}}$ the shell touches the inner rod at the point $D$. Once the pressure reaches some value $p_{\mathrm{e}}$, the shell touches the outer arbor at the point $A$. Generally, $p_{\mathrm{i}} \neq p_{\mathrm{e}}$ and the values $p_{\mathrm{i}}$ and $p_{\mathrm{e}}$ depend on the radii $R^{(i)}$ and $R^{(e)}$. Because of the symmetry of the problem, the part of the middle line $A B C D$ is sufficient to consider the state of the whole cross section. For the natural parameter $s$ the part $A B C D$ corresponds to $\left[s_{A}, s_{D}\right]=[0, \pi R / m]$.

For the analysis below it is convenient to use the dimensionless variables

$$
\begin{gathered}
s=R \theta, \quad \theta_{A}=0, \quad \theta_{D}=\frac{\pi}{m}, \\
\varkappa=R \tilde{\varkappa}, \\
\delta=\frac{R^{(i)}}{R}, \quad \xi=\frac{R^{(e)}}{R} .
\end{gathered}
$$

The angle $\varphi$ is measured off from the $O x$ axis and defines the arc length of the inner rod the part $C D$ of the cross section adjoins to. On one hand, the length of the part $C D$ has the form

$$
|C D|=\delta\left(\frac{\pi}{m}-\varphi\right)
$$

On other hand, the length of the part $C D$ can be expressed in terms of the parameter $\theta_{C}$

$$
|C D|=\frac{\pi}{m}-\theta_{C}
$$

These equations relate the parameter $\theta_{C}$ to $\varphi$ :

$$
\theta_{C}=\delta \varphi+(1-\delta) \frac{\pi}{m}
$$

As in the section 3 , the angle $\psi$ defines the arc of the outer arbor the part of the cross section $A B$ adjoins to, with $|A B|=\theta_{B}=\xi \psi$. In this case, for the phase $\Phi(\theta)$ Eq. (31) holds true as well. From 
the condition for the tangential vector to the surface of the shell section to coincide with the tangential vector to the surface of the rigid rod at the point $C$ we have

$$
\varphi=\Phi_{0}\left(\theta_{C}\right)-\Phi_{0}\left(\theta_{B}\right)+\psi
$$

The coordinates of the points of the free part $B C$ can be chosen in the form (see Fig. 10)

$$
x(\theta)=R \int_{\theta}^{\theta_{C}} \sin \Phi\left(\theta^{\prime}\right) \mathrm{d} \theta^{\prime}+R^{(i)} \cos \varphi, \quad y(\theta)=R \int_{\theta_{B}}^{\theta} \cos \Phi\left(\theta^{\prime}\right) \mathrm{d} \theta^{\prime}+R^{(e)} \sin \psi .
$$

At the point $C$ the ordinate of the inner rod coincides with the ordinate of the cross section:

$$
\int_{\theta_{B}}^{\theta_{C}} \cos \Phi\left(\theta^{\prime}\right) \mathrm{d} \theta^{\prime}+\xi \sin \psi=\delta \sin \varphi
$$

At the point $B$ the abscissa of the inner rod coincides with the abscissa of the cross section:

$$
\int_{\theta_{B}}^{\theta_{C}} \sin \Phi\left(\theta^{\prime}\right) \mathrm{d} \theta^{\prime}+\delta \cos \varphi=\xi \sin \psi
$$

At the point $C$ the external pressure is compensated by the reaction of the rod along the direction $\mathbf{n}$, hence for the shear force $q_{n}$ we have $q_{n}\left(\theta_{C}\right)=0$. Thus, taking into account (9) we conclude that the point $C$ minimizes the curvature:

$$
\varkappa\left(\theta_{C}\right)=a
$$

or

$$
\frac{4 K(k)}{\sqrt{A B}}=\theta_{C}
$$

Eqs. (39)-(42), (14) constitute a closed system of transcendent equations for the unknown parameters $a, b, a_{1}, \varphi, \psi$ as functions of the external pressure $p$, the number of the bulges $m$ and the radii $R^{(i)}$ and $R^{(e)}$. Fig. 11 shows a number of stages of the deformed shell. The left figure corresponds to the moment of touching the rod by the shell. This configuration satisfy Eqs. (36)-(38), (14). As the pressure $p$ is increased further, the shell envelops the rod and touches the outer arbor (the center figure). The third figure represents the limit state of the constrained shell. The second and third states satisfy Eqs. $(39)-(42),(14)$

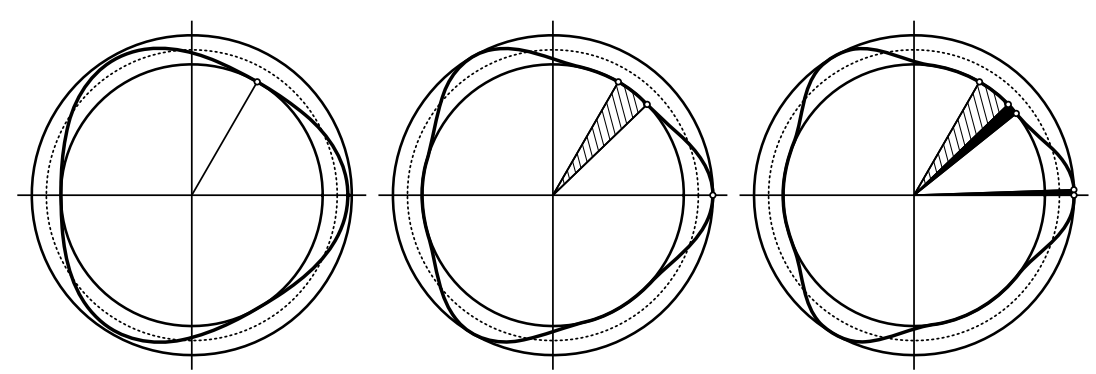

Figure 11. Changing in shape of the shell under two constraints $(\delta=0.9, \xi=1.1)$ : the left figure represents the moment when the shell touches the inner rod, the center figure represents the moment when the shell touches the outer arbor, the right figure is the limit state. The filled and hatched sectors denote increases of the angles relatively the previous states

Numerical analysis shows, that the deformation of the shell under the hydrostatic pressure can be steered with suitable choosing of the rigid constraints.

Fitting the radius of the outer arbor allows to limit the amplitude of the bulges of the deformed shell and prevent the rigid ribs from emerging on the shell surface. 


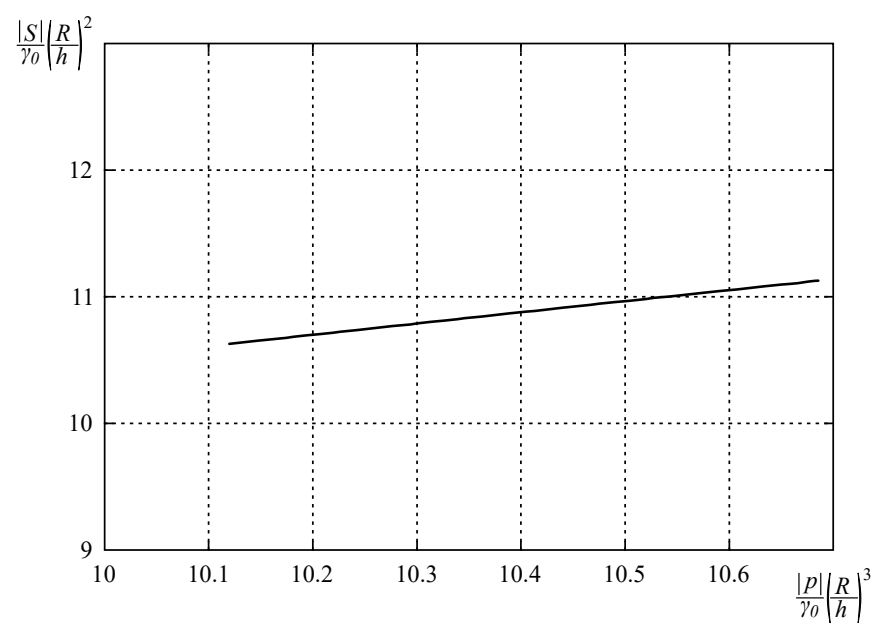

Figure 12. Dependence of the maximal intensity of the stress $S$ on the pressure for the shell under two constraints, $m=3, \delta=0.95, \xi=1.05$.

As the pressure is increased the internal stress concentrates nearby the smoothed crests of the bulges . This effect takes place within a narrower range of the pressure but is more considerable than for the shell being only under the outer arbor (Fig. 12). Once the stress surpass the yield strength of the material, the plastic flow will arise in the vertices of the crests. Then, the shell will get a new circular cross section, which will be retained on reducing the pressure. Changing the radii of the outer arbor and the inner rod along the axis allows to process a circular shell into an article of a variable diameter by the hydrostatic pressure, the material and time expenses being minimal.

Deformation of the shells made of low-carbon steel under such constraints was studied in the experiments of $[1,2]$. Comparatively thick metal shells for which $h / R \approx 0.12-0.2$ holds true were considered The length of every treated shell was 3-6 times greater the shell radius. During the experiments the shell always lost the stability of the shape, with $m=3$ bulges forming on the surface. Then, as the pressure was increased the shell enveloped the inner rod. When the difference between the radii of the shell and the rod was comparatively great, the ribs emerged on the shell surface. These ribs didn't reduce with further increase of the pressure. However, if the shell radius differed from the radius in the order $(2.2 .-2.6) h$, then the shell slipped along the rod arc under certain pressure and got the cross section similar to a square with smoothed vertices $(m=4)$. At the same time, the amplitude of the bulges decreased to a value of the order $(1.9-2.2) h$. For producing empty billets with circular cross section, an external arbor can be omitted when the amplitudes of the wave-like folds are not greater then $3 d$. The small amplitude folds are eliminated during the plastic flow of the material, when the internal stress reached the yield strength of the material at the crests of the bulges as the result of the pressure increase. However, when the perimeter length of the shell cross section differs significantly from the one of the arbor, rigid ribs arise on the shell surface. Then, a new approach is required to produce articles with smaller radii. Since coming the shell into contact with the external cylinder slows down the rate of the shell deformation as the pressure grows, introducing an additional external cylinder permits deliberate controlling the shell change in the form even when the magnitude of deformation is large. Ultimately, in $[1,2]$ the circular shell was processed into a specific hollow article, i.e. the steering head for a motorcycle, which has circular cross sections with different radii.

In this paper we neglected the thickness $h$ to simplify the analysis. Meanwhile, choosing the right relation between the shell thickness and the gap between the constraints is an important point for technological applications. Let us clarify the statement.

Let the amplitude of the bulges on the shell surface be equal $\zeta h: R^{(e)}=R+\zeta h$. The limit state of the shell that enveloped the rod has the folds with the thickness of the order $2 h$. The perimeters of the shell and the rod are to differ by the value

$$
2 \pi\left(R-R^{(i)}\right)=2 m h(\zeta-1) .
$$


Consequently, for $m=4, \zeta=2$ we find

$$
R \approx R^{(i)}+\frac{4 h}{\pi}, \quad R^{(e)} \approx R+2 h .
$$

\section{Methods of Numerical Calculations}

Numerical analysis can be simplified by the substitution

$$
a=-u+v ; \quad b=-u-v .
$$

Then, Eqs. (16), (23), (14) take the form

$$
\begin{gathered}
\pi \sqrt{A B}=4 K(k) m \\
\frac{-(A+B) u+(A-B) v}{A+B}+1+2 m[\Lambda(\beta, k)-1]=0 \\
\frac{8|p|}{\gamma_{0}}\left(\frac{R}{h}\right)^{3}=2 u\left(a_{1}^{2}+v^{2}\right) .
\end{gathered}
$$

Below we consider the coefficients $A, B, \beta$ and the modulus $k^{2}$ as functions of $u$, $v$. According to (46) the value $a_{1}^{2}$ can be expressed in terms of $u, v$ and $\tilde{p}=8|p| R^{3} /\left(\gamma_{0} h^{3}\right)$.

The reverse transform for Eq. (43) maps the domain of allowable parameters $\{(a, b): b<0, b<a<$ $\left.-b, 0 \leq k^{2} \leq 1\right\}$ in the first quadrant of the $u-v$ plane (Fig. 13). The shape of the domain stays the

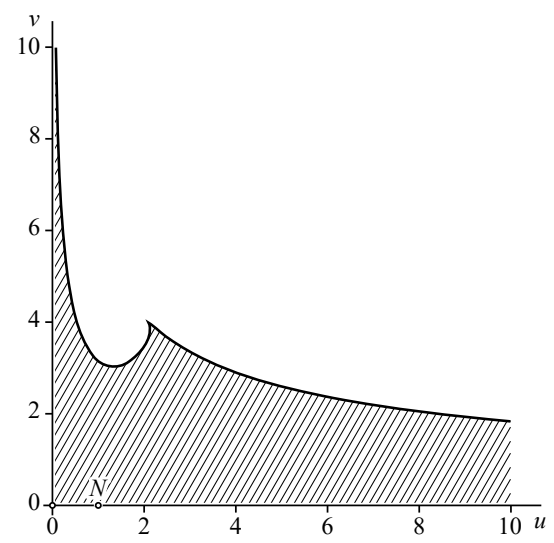

Figure 13. Domain of allowable values for $u, v$ at $\tilde{p}=8.5$.

same in qualitative terms at any allowable $\tilde{p}$.

We solved the system of Eqs. (44), (45) for $u, v$ with the Newton method. Here, the main issue is finding the initial values $u_{0}, v_{0}$ for the iterative algorithm. Let's note that the system (44), (45) is solvable for pressure $\tilde{p}_{\text {lin }}+\varepsilon$ for any natural number $m>2$, with $\tilde{p}_{\text {lin }}=m^{2}-1, \varepsilon \ll 1$. According to $(25)$ initial values for $u, v$ lie within a neighborhood of the point $N=(1,0)$ (Fig. 13). For a small enough $\Delta \tilde{p}$ resulting $u, v$ are a good initial approximation for the problem with the parameter $\tilde{p}_{\text {lin }}+\Delta \tilde{p}$. Iterating solution of the Eqs. (44), (45) with the parameter $\tilde{p}_{i}=\tilde{p}_{i-1}+\Delta \tilde{p}_{i}$ gives the initial approximation $u_{0}, v_{0}$ for any allowable pressure $\tilde{p}>\tilde{p}_{\text {lin }}$.

The problem (33), (34), (35), (14) for the shell constrained by the outer cylinder can be solved by the Newton method as well. However, we need two stages to find out the initial approximation $u_{0}, v_{0}$, $\psi_{0}$ for the iteration algorithm. At the first stage we determine the pressure $\tilde{p}_{T}$ at which the shell comes into contact with the outer cylinder. To do this, lets define the distance from the shell axis to the top of the shell bulge (Fig. 4):

$$
r_{A}=\left.\sqrt{x^{2}(\theta)+y^{2}(\theta)}\right|_{\theta=0} .
$$


According to (22), (24) the coordinates of the radius vector (1) are functions of $u, v$. Consequently $r_{A}$ is a function of $\tilde{p}$. In the outset of the shell deformation $r_{A}(\tilde{p})$ is monotonic, therefore we can determine the segment within which this function reaches the value $R^{(\mathrm{e})}$ by means of increasing the pressure by a suitable step $\Delta \tilde{p}$. We solve equation

$$
r_{A}(\tilde{p})=R^{(\mathrm{e})}
$$

by the bisection method and determine the pressure $\tilde{p}_{T}$ and corresponding solution $u_{\mathrm{T}}, v_{\mathrm{T}}$ for the system (44), (45). At $\tilde{p}=\tilde{p}_{\mathrm{T}}$ the parameters $u_{\mathrm{T}}, v_{\mathrm{T}}$ and $\psi_{\mathrm{T}}=0$ satisfy the system (33), (34), (35). At the second stage, we find the initial approximation $u_{0}, v_{0}, \psi_{0}$ for this problem using iteration $\tilde{p}_{i}=\tilde{p}_{i-1}+\Delta \tilde{p}_{i}$ beginning from the pressure $\tilde{p}_{\mathrm{T}}$.

For the problem of deforming the shell around the rigid rod, firstly, we find the pressure $\tilde{p}_{\mathrm{t}}$ at which the shell comes into contact with the inner rod in the point $B$ (Fig. 8) and the roots $u_{\mathrm{t}}, v_{\mathrm{t}}$ of the system of Eqs. (44), (45). The algorithm for searching for these parameters is similar to the case above. The values $u_{\mathrm{t}}, v_{\mathrm{t}}$ obtained and $\varphi_{t}=\pi / m$ satisfy the system $(36)-(38)$ at $\tilde{p}=\tilde{p}_{\mathrm{t}}$. Further, we find the initial approximation $u_{0}, v_{0}, \varphi_{0}$ for the problem with the parameter $\tilde{p}>\tilde{p}_{\mathrm{t}}$ using iteration over $\tilde{p}$.

The case of the shell subjected to two constraints (the outer hollow cylinder and internal rigid rod) is a combination of the former and latter ones. In the first place, the problem of coming the shell into contact with the rod is solved. Secondly, we use the solution of the problem of coming the shell into contact with the outer cylinder as the starting point for searching for the initial approximation for Eqs. (39)-(42) with a given parameter $\tilde{p}$.

\section{Conclusion}

In this paper, the influence of rigid constraints on the change in the shape of a shell subjected to the hydrostatic pressure is studied. It is also shown that it is feasible to obtain the state of the shell with a variable diameter along the generating line within a finite range of hydrostatic pressures. The use of an additional outer cylinder turns out to be fundamentally important. Limiting the amplitude of bulges by means of the cylinder allows one to avoid the emergence of rigid ribs on the shell surface. Besides, the external constraint leads to the concentration of internal stresses near the tops of the bulges. As a consequence, the external pressure value at which the shell takes a new circular shape can be decreased in tens times. Using simple examples we analyzed the general patterns of technology for producing the circular cylindrical shells into hollow articles of complex shape by the method of hydrostatic compression with minimal time, energy and material costs.

Acknowledgements. The authors would like to thank B.I. Kamenetsky for his attention to the work and many interesting discussions and A.A. Rascovalov for a technical assistance.

This work was performed as a part of the state program of the Federal Agency for Scientific Organizations (the theme "Quantum", No 01201463332 (AAAA-A18-118020190095-4)).

\section{References}

1. B.I. Kamenetsky, H.H. Talutz and V.A. Obukhov, "Studing the process of plastic deformation of cylindrical metall shells with highly pressurized liquid," Physics of Metals and Metallography, vol. 81, no.3, pp.159-167, 1996. (In Russian)

2. B.I. Kamenetsky, V.V. Kiselev. "Studying processes of plastic deformation of cylindrical shells with high pressure liquid," Smith and stamping production, no.7, pp.11-17, 2007. (In Russian)

3. A.S. Vol'mir, Stability of Elastic Systems. Foreign Technology Division, Air Force Systems Command, WrightPatterson Air Force Base, 1965.

4. J.M.T. Thompson, G.W. Hunt, Instabilities and Catastrophes in Science and Engineering. Wiley, 1982.

5. Y. Zhu, X.Y. Luo and R.W. Ogden, "Nonlinear axisymmetric deformations of an elastic elastic tube under external pressure," European Journal of Mechanics A/Solids vol.29, pp.216-229, 2010.

6. Y. Zhu, X.Y. Luo, H.M. Wang, R.W. Ogden and C. Berry, "Three-dimensional non-linear buckling of thickwalled elastic tubes under pressure," Int. J. Non-linear Mech., vol.48, pp.1-14, 2013.

7. F.C. Bardy, S. Kyriakides, H.D. Yun, "Plastic buckling of circular tubes under axial compression - part II: Analysis," International Journal of Mechanical Sciences, vol.24, pp.842-854, 2006. 
8. V.L. Yakushev, Nonlinear deformations and stability of thing shells. Nauka, 2004. (In Russian)

9. M.A. Lavrentyev, A.Y. Ishlinsky, "Dynamical forms of the elastic systems stability loss," Proceedings of the USSR Academy of Sciences, vol.64, no.6, pp.779-782, 1949. (In Russian)

10. R. Frisch-Fay, Flexible Bars, Butterworths, 1962.

11. C.H. Wu, R. Plunkett, "On the Contact Problem of Thin Circular Rings." J. Appl. Mech., vol.32, no.1, pp.11-20, 1965.

12. G. Domokos, W.B. Fraser, I. Szebere'nyi, "Symmetry-breaking bifurcations of the uplifted elastic strip," Physica D, vol.185, pp.67-77, 2003.

13. D.V. Dolgikh, V.V. Kiselev, "Corrugating of a flexible ring under an external hydrostatic pressure," J. Appl. Math. Mech, vol.74, pp.204-213, 2010.

14. V.V. Kiselev, D.V. Dolgikh, Non-linear patterns of dents on surfaces of plates and shells under load. Physmatlit, 2012. (In Russian)

15. S.S. Antman, Nonlinear problems of elasticity. Springer-Verlag, 1995.

16. R. Hill, The Mathematical Theory of Plasticity. Clarendon press, 1998.

17. H. Hodges Deway, "Non-linear in plane deformation and buckling of rings and high arches," Int. J. Non-linear Mech. vol.34, pp.723-737, 1999.

18. P.F. Byrd, M.D. Friedman, Handbook of elliptic integrals for engineers and scientists. Springer, 1971. 